\title{
Características dos Três Mundos da Matemática QUE EMERGEM NA RESOLUÇÃO DE QUESTÕES ENVOLVENDO O CONCEITO DE LIMITE ${ }^{1}$
}

\author{
CHARACTERISTICS OF THE THREE WORLDS OF MATHEMATICS THAT \\ EMERGE IN SOLVING QUESTIONS INVOLVING THE CONCEPT OF LIMIT
}

DOI: http://dx.doi.org/10.23926/RPD.2526-2149.2019.v4.n1.p80-95.id370

\author{
Gabriel de Oliveira \\ Soares \\ Doutorando em Ensino de \\ Ciências e Matemática pela \\ Universidade Franciscana \\ (UFN) \\ gsoares8@outlook.com
}

\begin{abstract}
Resumo: O conceito de limite de uma função é concebido com um dos principais e mais complexos no trabalho com as disciplinas de Cálculo Diferencial e Integral (CDI), sendo base para a aprendizagem do conceito de derivada, por exemplo. Nesse sentido, buscando compreender como alunos que já cursaram uma disciplina de CDI compreendem esse conceito, esse estudo objetiva analisar características dos Três Mundos da Matemática propostos por Tall (2004; 2013) que estudantes de dois cursos de Licenciatura em Matemática mobilizam ao resolverem questões envolvendo o conceito de limite. Para tal, foi proposto um teste com sete questões à catorze estudantes de dois cursos de Licenciatura em Matemática do estado do Rio Grande do Sul, das quais, três são analisadas nesse texto, com vistas ao quadro teórico proposto. Pode-se concluir que a maioria dos estudantes apresenta imagens conceituais fracas em relação a esse conceito, estando suas aprendizagens baseadas principalmente em características do Mundo Conceitual Corporificado.
\end{abstract}

Palavras-chave: Educação Matemática no Ensino Superior; Limite de uma função; Três Mundos da Matemática.

\begin{abstract}
The concept of limit of a function is conceived with one of the main, and also one of the most complex in the work with the disciplines of Differential and Integral Calculus (CDI), being the basis for learning the concept of derivative, for example. In this sense, in order to understand how students who have already studied a CDI discipline understand this concept, this study aims to analyze the characteristics of the Three Worlds of Mathematics proposed by Tall $(2004 ; 2013)$ that students of two Mathematics Degree courses mobilize when solving questions involving the concept of limit. For that, a test with seven questions was proposed to the fourteen students of two courses of Mathematics Degree of the state of Rio Grande do Sul, of which, three are analyzed in this text, in view of the proposed theoretical framework. It can be concluded that most of the students present weak conceptual images in relation to this concept, being their learning based mainly on the characteristics of Conceptual Embodied World.
\end{abstract}

Keywords: Mathematics Education in Higher Education; Limit of a function; Three Worlds of Mathematics.

\footnotetext{
${ }^{1}$ O presente trabalho foi realizado com apoio da Coordenação de Aperfeiçoamento de Pessoal de Nível Superior - Brasil (CAPES) - Código de Financiamento 001.
} 


\section{INTRODUÇÃO}

É de conhecimento comum no âmbito acadêmico, a atual situação do ensino de Cálculo no Ensino Superior. Estudos como os de Lopes (1999), Sena e Souza (2015) e Staron (2016) evidenciam índices de reprovação de até $70 \%$ nas disciplinas deste componente curricular nos cursos superiores.

O reflexo deste alto índice de reprovação pode ser constatado em diversas universidades do país: cada vez tem-se mais turmas nas disciplinas de Cálculo compostas, em grande parte, por estudantes que já deveriam ter obtido aprovação.

Além disso, todas essas reprovações podem acabar levando os estudantes à evasão, como destacado por Meyer e Souza Júnior (2002), ao afirmarem que há inúmeras reclamações de alunos ou de professores de outras áreas acerca da inexistência de esforços para tornar Cálculo interessante ou útil.

Levando em consideração esses fatores, e refletindo sobre os conceitos matemáticos abordados nestas disciplinas, além do apontado por Artigue (1995) - ao dizer que uma das principais causas da complexidade do Cálculo se dá também por dificuldades associadas à conceitualização e à formalização da noção de limite, centro do campo do cálculo - , realizouse um estudo com alunos de cursos de formação inicial de professores de matemática, a fim de verificar como estes compreendem o conceito de limite.

Para tanto, utilizou-se como base teórica a Teoria dos Três Mundos da Matemática, proposta por David Tall $(2004 ; 2013)$, um quadro teórico que busca explicar a aprendizagem em matemática por três linhas de pensamento, chamadas por ele de mundos: o Mundo Conceitual Corporificado, o Mundo Proceitual Simbólico e o Mundo Axiomático Formal.

Dessa forma, o objetivo deste trabalho é analisar as características dos Três Mundos da Matemática (TALL, 2004; 2013), que estudantes de dois cursos de Licenciatura em Matemática mobilizam ao resolverem questões envolvendo o conceito de limite de uma função. Para isso, apresenta-se brevemente a literatura sobre o ensino de Cálculo e a teoria que dá suporte ao trabalho. São também apresentados os procedimentos metodológicos da pesquisa e seus resultados.

\section{Três Mundos da Matemática}

David Tall iniciou sua trajetória de pesquisa na área de Matemática, concluindo seu primeiro doutoramento no ano de 1967. Mas, no desenvolvimento de seu percurso profissional, 
percebeu que se interessava pela área da Educação Matemática, mais especificamente, pela Psicologia da Educação Matemática, e fez sua "transição" para essa área.

Em 1986, Tall defendeu sua tese em Educação Matemática, intitulada Building and Testing a Cognitive Approach to the Calculus Using Interactive Computer Graphics, desenvolvida sob a orientação de Richard Skemp e voltada para a aprendizagem de Cálculo Diferencial e Integral com a utilização do computador.

Nesta tese, Tall apresentou alguns estudos voltados à Psicologia da Educação Matemática, revisando as ideias de Piaget, Dienes e Skemp, e formulou uma atividade de ensino baseada na abordagem cognitiva para o estudo do Cálculo.

Nesse período de transição da área da Matemática para a Educação Matemática, Tall conheceu o pesquisador israelense Shlomo Vinner, com quem veio a trabalhar em uma das suas principais contribuições teóricas à Educação Matemática: as ideias de Imagem Conceitual e Definição Conceitual ${ }^{2}$.

Pesquisando nessa área desde então, em 2004, Tall publicou seus primeiros escritos sobre a Teoria dos Três Mundos da Matemática, tomando como base algumas reflexões de Jean Piaget, Jerome Bruner, Efraim Fischbein e Pierre van Hiele, além de suas próprias pesquisas anteriores.

Enquanto quadro teórico, Tall apresenta três "mundos" da Matemática, nos quais a aprendizagem se desenvolve:

a) O Mundo Conceitual Corporificado, ou apenas Mundo Corporificado, "diz respeito às percepções acerca do mundo e o pensamento a respeito das coisas que são percebidas e sentidas não apenas no mundo físico, mas em um mundo mental de significados" (TALL, 2004, p. 2). Assim, o pensamento matemático que está ligado a este mundo baseia-se em relações com objetos corporificados, como gráficos e tabelas.

b) O Mundo Proceitual Simbólico, ou Mundo Simbólico, é aquele no qual os símbolos matemáticos são usados não só para representar e efetuar ações, mas também para representar o produto que é resultado dessas ações. Tall $(2004$, p. 3) destaca que "as atividades neste mundo se iniciam com ações (como apontar e contar) e são incorporadas como conceitos por meio do uso de símbolos".

c) O Mundo Formal Axiomático, ou Mundo Formal, conforme Tall (2004, p. 3), “é baseado em propriedades, expressas em termos de definições formais que são usadas como

\footnotetext{
2 "Concept image" e "concept definition". Esses termos também têm sido traduzidos por "imagem de conceito e definição de conceito" ou, ainda, "conceito imagem e conceito definição".
} 
axiomas para especificar as estruturas matemáticas (por exemplo, 'grupo', 'campo', 'espaço vetorial' e 'espaço topológico' e assim por diante)".

Dessa forma, uma pessoa que aprende matemática inicia sua trajetória de aprendizagem em um dos mundos mais elementares, nos níveis de matemática prática, a saber, os Mundos Corporificado e Simbólico, e pode chegar a aprender os "conceitos cristalinos" da matemática, ao elevar suas aprendizagens ao Mundo Formal.

Logo, ao buscar as recursos que estudantes de cursos de Licenciatura em Matemática mobilizam ao resolverem questões envolvendo o conceito de limite de uma função, busca-se compreender como se deu a aprendizagem desse conceito por parte dos estudantes, e como o trabalho em sala de aula pode melhorar, a fim de formar imagens conceituais mais consolidadas.

\section{Desafios no Ensino do CálCUlo na Formação de PROFESSORES}

Ao discutir o ensino das disciplinas de Cálculo, em especial nos cursos de Licenciatura em Matemática, é necessário inicialmente apontar o que trazem as Diretrizes Curriculares Nacionais para os Cursos de Licenciatura em Matemática, no que tange à organização dos conteúdos curriculares. De acordo com este documento (BRASIL, 2001, p. 4, grifo do autor),

Os conteúdos curriculares dos cursos de Matemática deverão ser estruturados de modo a contemplar, em sua composição, as seguintes orientações:

a) partir das representações que os alunos possuem dos conceitos matemáticos e dos processos escolares para organizar o desenvolvimento das abordagens durante o curso;

b) construir uma visão global dos conteúdos de maneira teoricamente significativa para o aluno.

Desta forma, nas disciplinas de Cálculo, assim como nas outras disciplinas de cunho específico da formação do professor de Matemática, os conteúdos curriculares devem ser apresentados de maneira ampla, com uma abordagem que possibilite estabelecer relações entre os conteúdos, aplicações e a prática docente, a fim de garantir uma aprendizagem significativa. Essa abordagem também deve ser visualizada em outros cursos superiores, levando em consideração a aprendizagem como processo primordial no meio educacional. Entretanto, isso não vem acontecendo de maneira natural.

Frota (2001, p. 91) destaca que o ensino da Matemática precisa - urgentemente libertar-se das amarras de um ensino passo a passo, que não incentiva o conhecimento matemático relacional, para possibilitar que o estudante estabeleça relações entre os vários conceitos estudados. 
Ainda sobre esta questão e, especialmente, acerca do tratamento de tópicos matemáticos como procedimentos, Barufi (1999, p. 162) afirma que

A fim de minimizar o insucesso na construção do conhecimento significativo, a saída, muitas vezes adotada, é a de privilegiar a aplicação do cálculo, apresentando um grande número de problemas e exercícios, muitas vezes repetitivos, onde o aluno acaba memorizando, de alguma forma, processos de resolução. Nesse sentido, reduzse a ideia, o conceito, ao algoritmo e sobra aquela eterna pergunta dos estudantes, não respondida e "odiada" pelos professores: Pra que serve isto?

Corroborando o apresentado, e pensando especificamente na formação de professores de matemática, é importante que se tenha em mente ainda que, uma formação na qual se objetiva desenvolver a criação de uma postura docente por parte dos alunos - o caso da Licenciatura em Matemática - deve oportunizar a compreensão de elementos que caracterizem também os processos de ensino e aprendizagem das questões do Cálculo Diferencial e Integral.

Entretanto, segundo Cury e Bazzo (2001), os docentes que lecionam disciplinas matemáticas em cursos da área de ciências exatas são, em sua maioria, licenciados ou bacharéis em Matemática, com pós-graduação em Matemática Pura ou Aplicada, o que os pode relacionar com o desenvolvimento de uma postura em que há a necessidade de desenvolver rigor matemático nas disciplinas de cunho específico.

Além disso, volta-se às discussões do começo desta sessão: as Diretrizes Curriculares Nacionais para os Cursos de Licenciatura em Matemática afirmam que se deve ter por objetivo, ao trabalhar os conceitos matemáticos, construir uma visão global dos conteúdos de maneira teoricamente significativa para o aluno.

Mas, até que ponto isto verdadeiramente ocorre? Cury e Bazzo (2001) afirmam que, nos cursos de formação de professores, a Matemática apresenta-se isolada dos outros conteúdos e especialmente do mundo em que estão inseridos os alunos, futuros professores. Ainda, Cury e Bazzo (2001, p. 34) citam que,

Os professores universitários de Matemática não fogem a essa classificação de Vasconcelos. Muitas vezes, suas atuações nos cursos de licenciatura ou nas engenharias vêm, ainda, matizadas pela posição elitista dessa ciência, que faz com que considerem "perda de tempo" discutir quaisquer assuntos que fujam da apresentação "burocrática" dos conteúdos programáticos. Quando solicitados a discutir reformulações curriculares, por exemplo, esses professores evitam se expor, pois, em geral, sua formação matemática teve os mesmos problemas citados, ou seja, eles não aprofundaram as aplicações dessa ciência às outras áreas, não discutiram as consequências do uso das ferramentas matemáticas, apenas aprenderam a usá-las, com muita propriedade, com muitos detalhes, mas absolutamente descontextualizadas.

Dessa forma, a relação do Cálculo com outras disciplinas e conteúdos programáticos, e até mesmo com aspectos da realidade, pode vir a ser ignorada no processo de ensino. Esses são alguns dos desafios encontrados no ensino do Cálculo nos cursos de formação de professores 
de Matemática que necessitam ser discutidos em trabalhos que abordem o ensino de Cálculo no Ensino Superior.

\section{Procedimentos metodológicos}

Esse trabalho é um recorte de uma pesquisa de mestrado que tem por objetivo analisar o conceito de limite de uma função apresentado por estudantes de dois cursos de Licenciatura em Matemática, bem como suas estratégias de resolução de questões, à luz da Teoria dos Três Mundos da Matemática.

A fim de atender o objetivo da pesquisa, foi aplicado um teste a catorze estudantes de dois cursos de Licenciatura em Matemática de duas instituições de Ensino Superior do estado do Rio Grande do Sul. A amostra foi selecionada por conveniência, sendo pré-requisito para responder o teste que, até o momento da aplicação, os alunos houvessem cursado ao menos uma disciplina de Cálculo em sua formação. O teste continha sete questões, das quais três são analisadas nesse texto.

A partir do teste, trabalhou-se com uma análise qualitativa dos dados, visando analisar as características dos Mundos da Matemática propostos por Tall, que eram verificadas nas resoluções das questões dos estudantes. Além disso, as respostas foram analisadas da seguinte forma: inicialmente, foram classificadas em corretas, parcialmente corretas, incorretas ou em branco. Após, foi feito um levantamento do tipo das respostas dos estudantes, e assim foi feita uma classificação, criada a partir de uma análise comparativa proveniente do tratamento dos dados realizada em outros trabalhos que utilizaram o mesmo quadro teórico, revisados em outro trabalho (SOARES; CURY, 2017a).

Em seguida, foi realizada a análise a partir do quadro teórico dos Três Mundos da Matemática, observando as características desses mundos que emergiram na resposta dos estudantes para cada questão.

Para evitar identificação, as respostas dos alunos foram indicadas por A1, A2, ..., A14. Nos casos em que a resposta não estava legível, optou-se por digitá-la, conservando a linguagem da forma como foi enunciada pelo licenciando; em outros casos, foi digitalizada a resposta, para ser fiel à figura desenhada pelo respondente.

\section{APRESENTAÇÃo E ANÁliSE dOS DADOS}

A primeira questão proposta aos estudantes para que resolvessem foi a seguinte:

Questão 1. Para a função abaixo definida, estime o limite no ponto zero, se for possível, calculando a função nos pontos indicados: 


$$
f(x)=\frac{\sqrt{x+1}-1}{x}, \text { nos pontos } x= \pm 0,25 ; \pm 0,1 ; \pm 0,01 ; \pm 0,001
$$

Após, confirme suas conclusões sobre o limite esboçando o gráfico da função em um intervalo adequado.

Esse tipo de questão é bastante comum em livros didáticos de cálculo e foi adaptada do livro de Anton, Bivens e Davis (2007). Esperava-se que os estudantes calculassem o valor do limite para os pontos dados e após estimassem o valor do limite para $x=0$. Além disso, solicitou-se que os alunos esboçassem o gráfico da função para um intervalo adequado. É importante destacar aqui que não se esperava que os estudantes fizessem um estudo de assíntotas, pontos de máximo e mínimo e outros fatores que podem ser visualizados por caminhos do Cálculo, mas sim, que esboçassem o gráfico com base apenas na visualização dos valores da função nos pontos dados.

Os alunos A4, A7, A10 deixaram a questão sem resposta. As respostas dos demais estudantes são analisadas no que segue.

Os estudantes A1, A2, A3 e A6 apresentaram assertivas condizentes com o problema proposto e com o que se esperava como resposta, classificadas como corretas. O gráfico de A3 é o que mais se aproxima do gráfico da função dada, enquanto que o gráfico de A2 apresentou certos problemas de escala e os de A1 e A6 necessitam um olhar para os números negativos. Como exemplo, apresenta-se a resposta de A3 na Figura 1. Percebe-se que há um bom desenvolvimento, com as características do Mundo Simbólico apresentadas pelos três estudantes e do Mundo Conceitual Corporificado, especialmente por A3. 
Figura 1 - Resposta de A3 à primeira questão

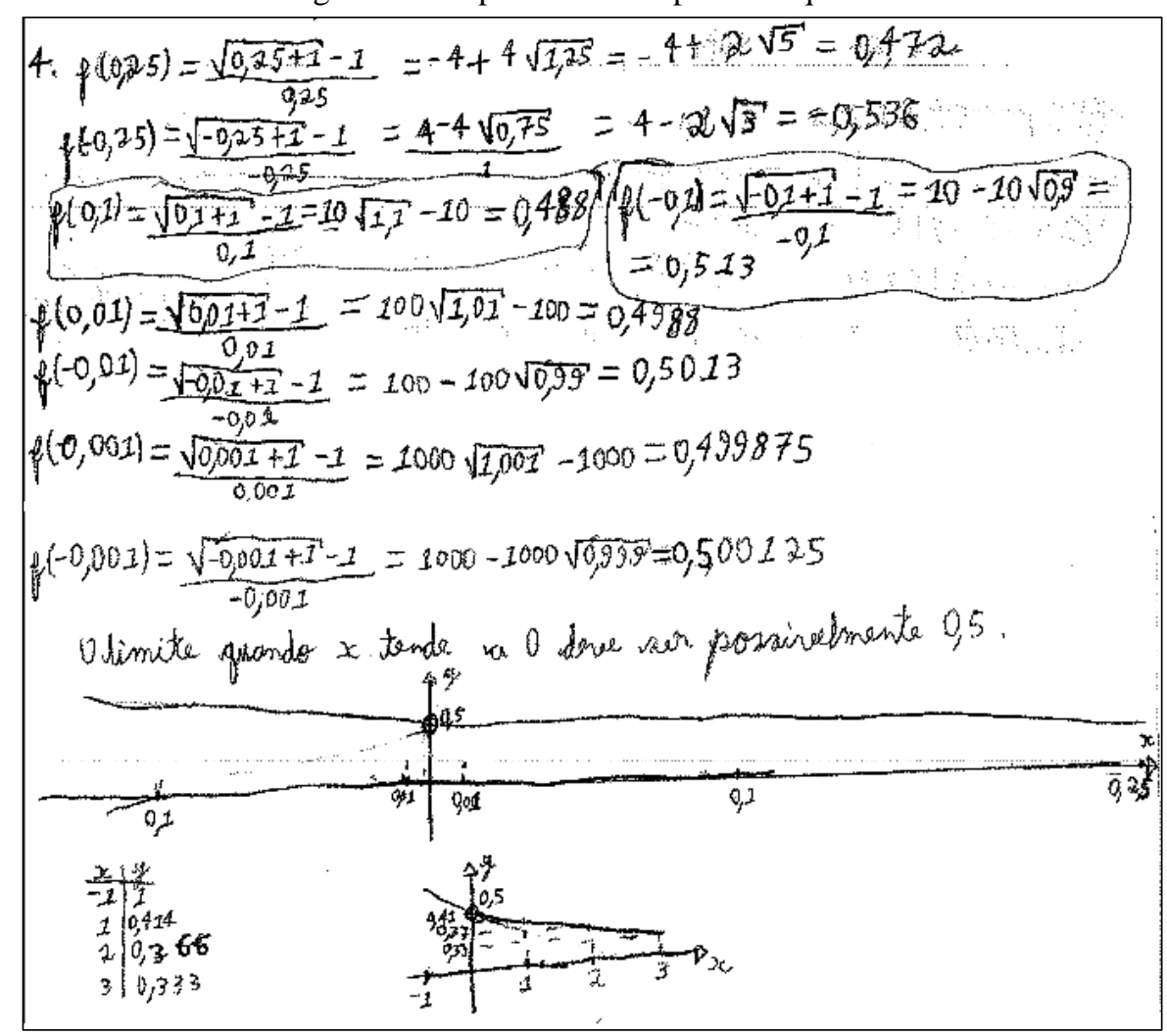

Fonte: Dados da pesquisa

A resposta dada por A5 foi considerada parcialmente correta, pois o aluno não concluiu o valor do limite para os pontos e nem calculou o valor da função para todos os pontos dados. Dito isso, destaca-se características dos Mundos Simbólico e Corporificado. Esta é apresentada na Figura 2.

Figura 2 - Resposta de A5 à primeira questão

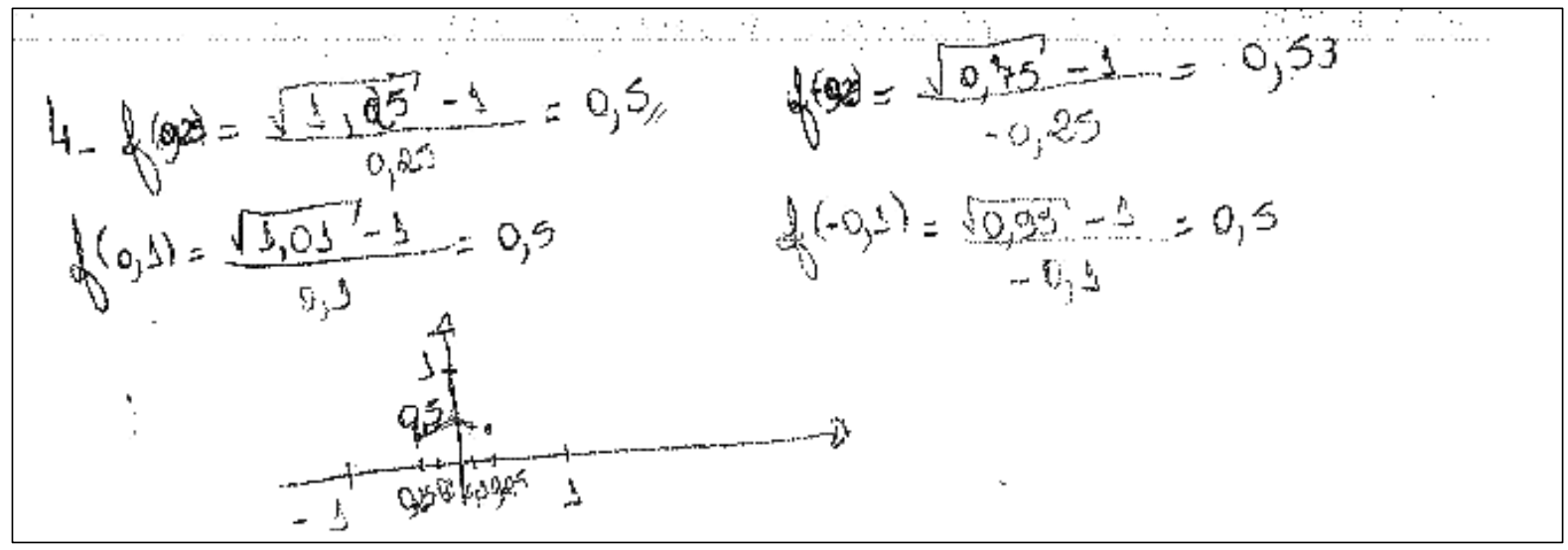

Fonte: Dados da pesquisa

Já a resposta de A8 (Figura 3) foi classificada como incorreta, pois o aluno calcula os valores para os pontos dados, mas não consegue perceber as aproximações pelos limites, ou percebe-os, mas acredita que os valores sejam diferentes, e conclui que não existe limite. 
Percebe-se a dificuldade em compreender os infinitésimos, já elencada por Zuchi e Gonçalves (2003), o que reitera a visão dos autores. Em relação aos Mundos da Matemática, percebe-se características do desenvolvimento dos Mundos Conceitual Corporificado e Proceitual Simbólico, ao construir o gráfico e realizar os cálculos dos limites nos pontos dados.

Figura 3 - Resposta de A8 à primeira questão

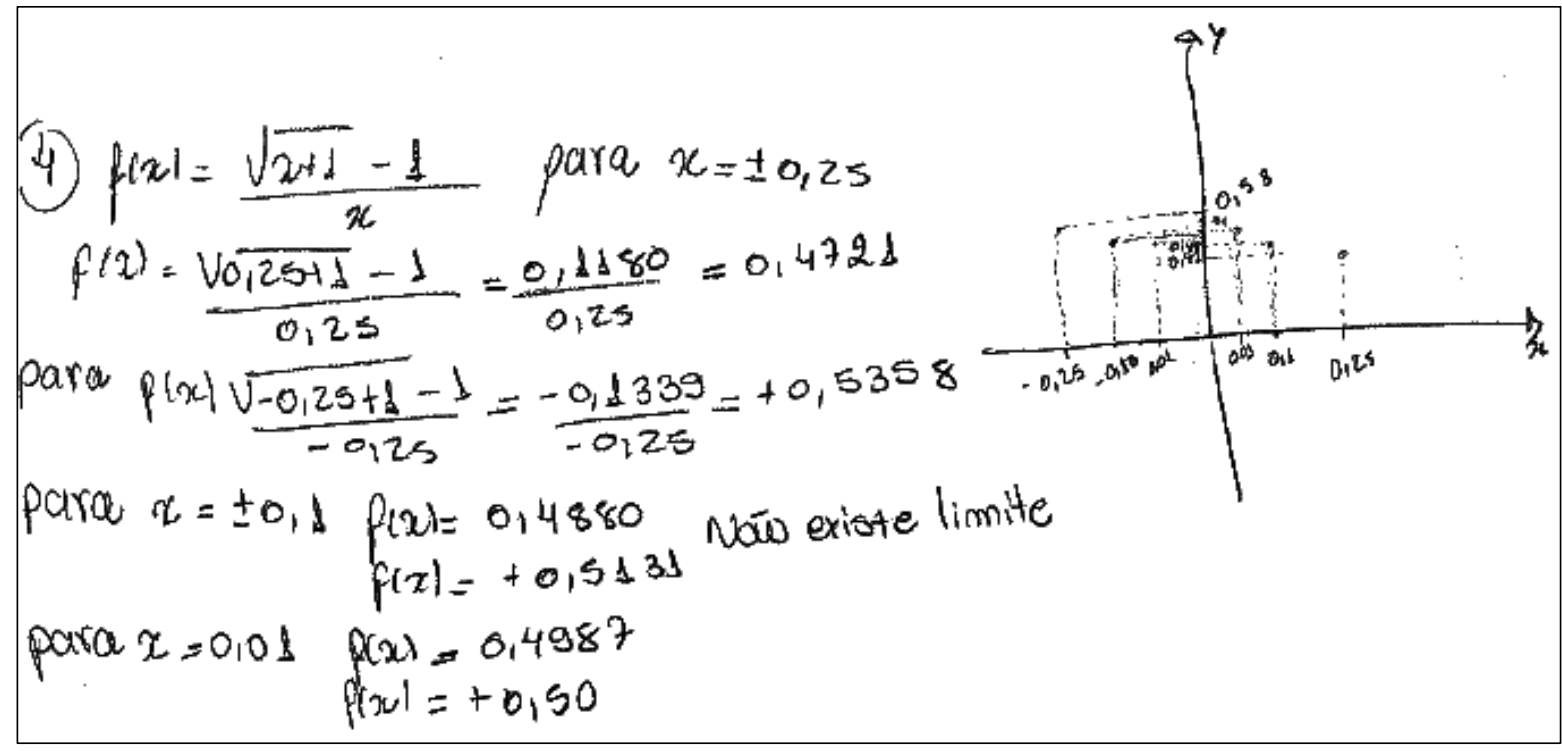

Fonte: Dados da pesquisa

É apresentado um recorte da resposta de A9, pois o aluno desenvolveu o cálculo em três páginas, mas, no geral, toda a escrita se assemelha ao que foi mostrado na Figura 4. Sua explicação foi classificada como parcialmente correta, porque o aluno não consegue afirmar o valor do limite ao final do cálculo. Há uma característica interessante que é essa articulação dos números decimais como fração decimal, mas ao final, ele não consegue esboçar o gráfico da função nem afirmar o limite dado. Há apenas características do Mundo Simbólico em sua resposta.

Já as respostas de A11 e A12 foram classificadas como corretas, pois os alunos desenvolvem adequadamente o que se esperava como resposta para essa questão. Ficaram evidenciadas características dos Mundos Corporificado e Simbólico por ambos os estudantes. Como exemplo, apresenta-se a resposta de A12 na Figura 5. 
Figura 4 - Recorte da resposta de A9 à primeira questão

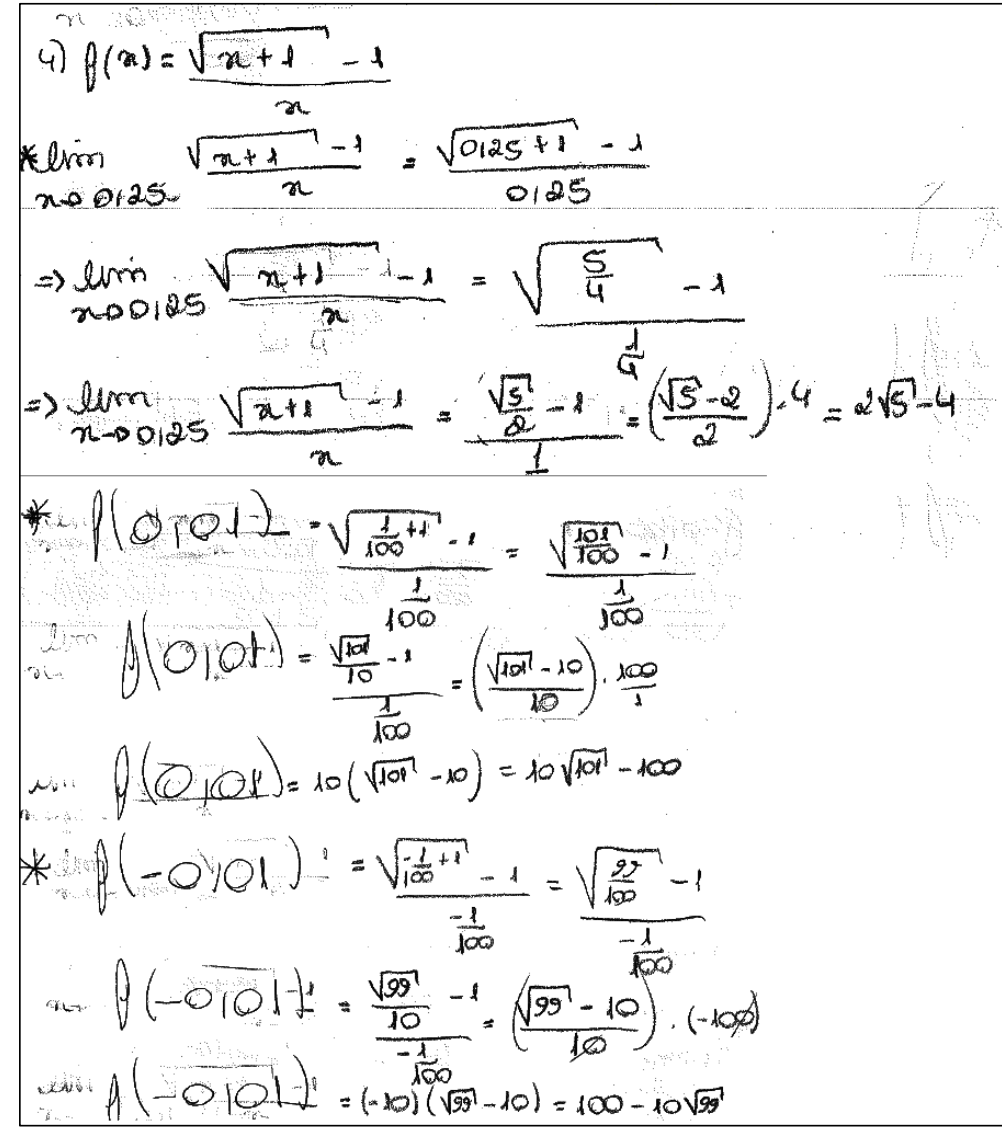

Fonte: Dados da pesquisa

Figura 5 - Resposta de A12 à primeira questão

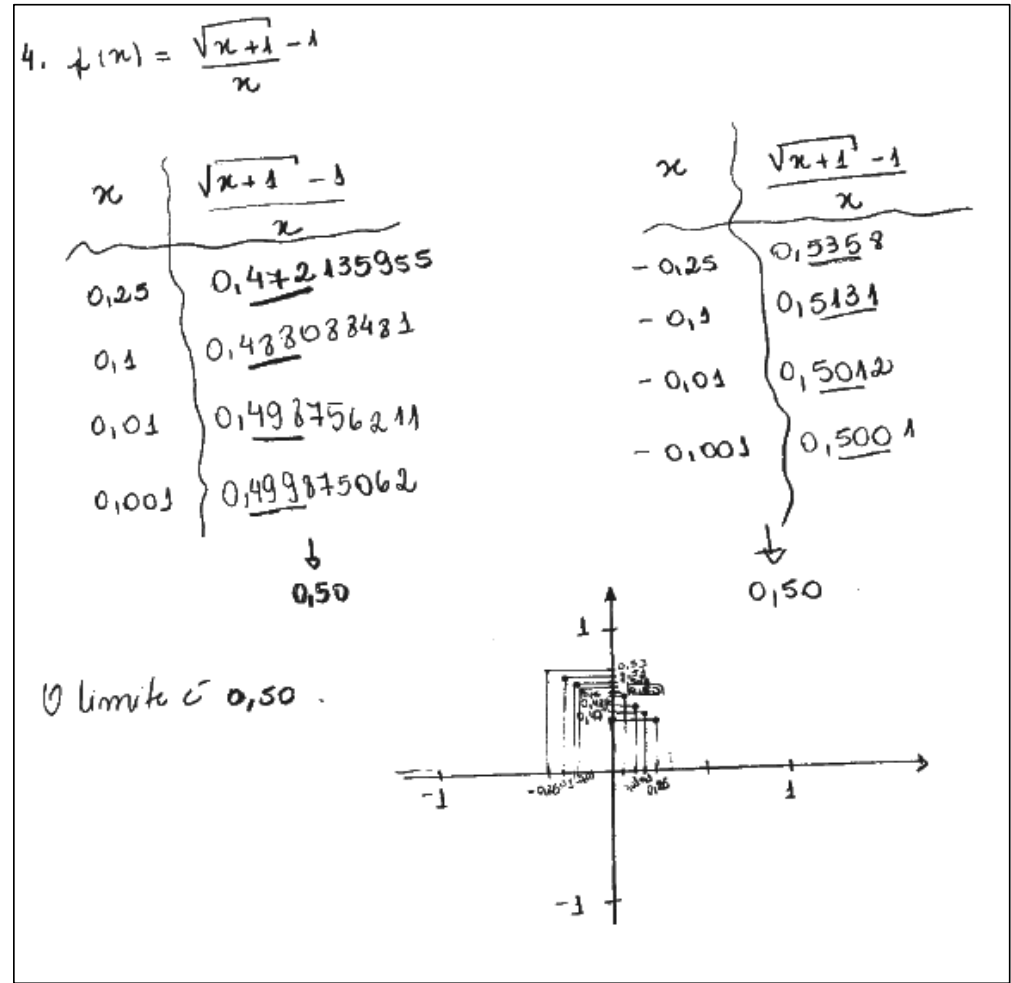

Fonte: Dados da pesquisa 
A segunda questão, adaptada de Anton, Bivens e Davis (2007), propunha o seguinte:

Questão 2. Considere a função $f$ cujo gráfico é representado abaixo. Para que valores existe $\lim _{x \rightarrow x_{0}} f(x)$ com $-9 \leq x_{0} \leq 4$ ? Justifique sua resposta.

Figura 6 - Gráfico da questão 2

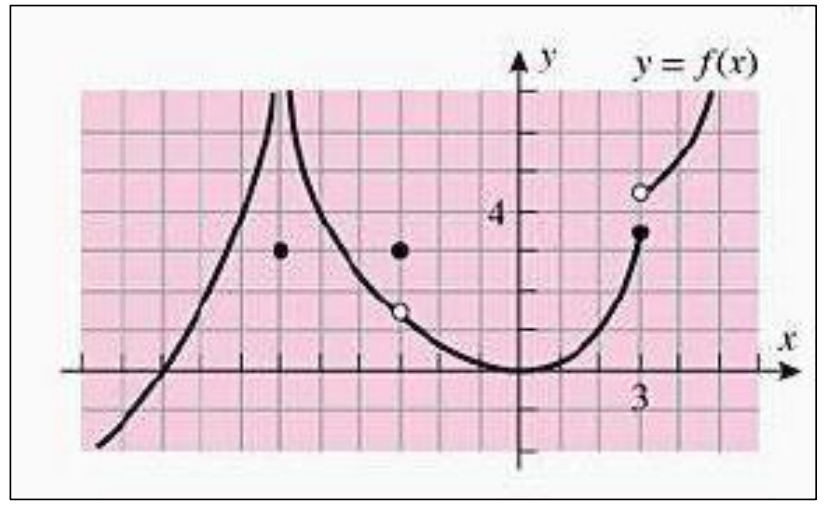

Fonte: Anton, Bivens e Davis (2007).

Essa questão apresentada aos estudantes trabalha com ideias do Mundo Conceitual Corporificado para identificar a existência do limite no domínio dado, mais especificamente, nos pontos destacados no gráfico.

Esperava-se, como resposta a esta questão, que os alunos apontassem a inexistência do limite nos pontos -6 e 3, para o domínio dado. Essa questão também discute as relações de continuidade e existência de limite e, por isso, acredita-se que é uma questão válida para esse trabalho. Sete estudantes deixaram a questão em branco, sendo eles A5, A8, A10, A11, A12, A13 e A14. A seguir, apresentam-se as respostas dos demais alunos.

A1: Existe limite para $-9 \leq x_{0} \leq 4$, pois para qualquer valor que atribuirmos para $x$ teremos um valor em y.

A resposta de A1 foi classificada como incorreta, pois o aluno não discutiu as relações da existência do limite nos pontos em dúvida no gráfico. Além disso, percebe-se que, para responder, o aluno somente utilizou uma justificativa baseada na observação gráfica, o que remete a características do Mundo Conceitual Corporificado.

A2: Nota-se, pelo gráfico que essa função não é contínua, ou seja, há saltos/falhas em sua representação. Logo, os valores de x para que o limite existe são $[-9,-3[,[-3,3]$.

A resposta de A2 foi classificada como incorreta, pois mesmo que discuta as ideias de existência de limite, o faz de maneira incorreta. Fica bem claro também que, ao expressar sua resposta, só são apresentadas características do Mundo Conceitual Corporificado, por mobilizar apenas a questão da visualização gráfica na escrita. 
As respostas de A3, A4 e A6 (Figura 7) foram classificadas como parcialmente corretas, pois os alunos apontaram a inexistência do limite em um dos dois pontos. É interessante destacar nesta discussão a questão da existência desse limite infinito. Muitos estudantes consideram que o símbolo do infinito é um numeral, uma quantidade infinita. Mas essa é uma

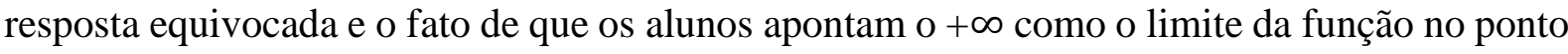
$x_{0}=-6$ pode ser considerado como um já-encontrado que prejudica a aprendizagem. Ademais, destaca-se que os alunos apresentaram características do Mundo Corporificado em sua resposta.

A3: $\lim _{x \rightarrow x_{0}} f(x)$ existe em todos os $x \neq 3$, pois $x=3$ é o único valor em que não há limites laterais iguais, enquanto em $x=-3$ estes valores existem, ela apenas não é contínua, valendo o mesmo para $x=-6$, onde o limite é $+\infty$. Resposta: $A=\left\{x_{0} \mid x_{0} \neq 3\right\}$.

Figura 7 - Resposta de A6 à segunda questão

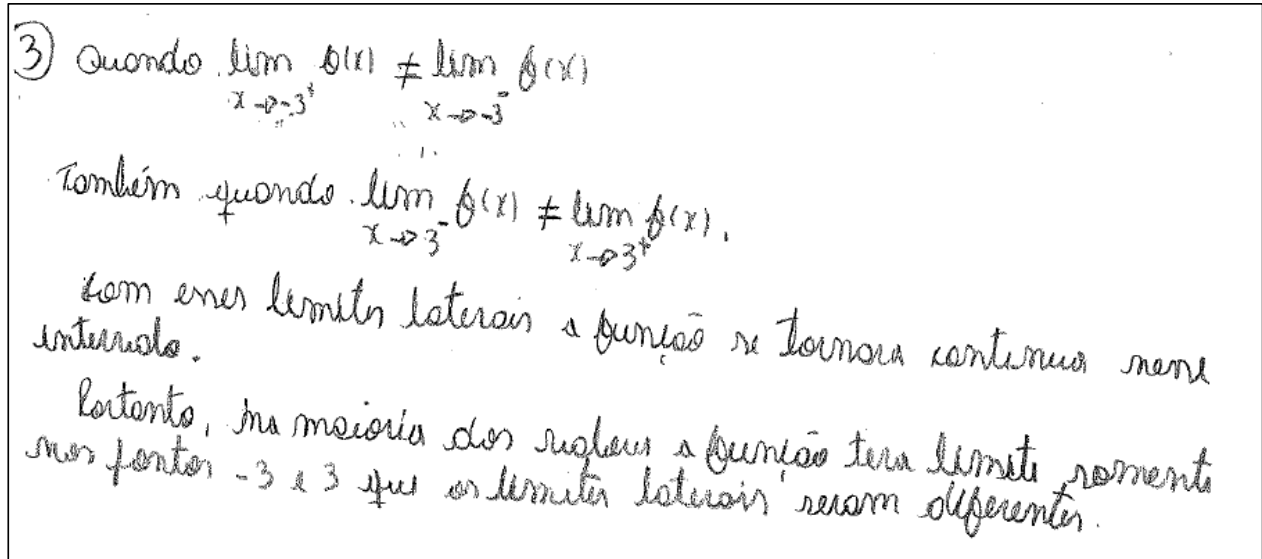

Fonte: Dados da pesquisa

A7: $-8,-7,-6,-5,-5,-2,-1,1,2$ tem limite.

A7 teve sua resposta classificada como incorreta, pois ignorou o intervalo real e pensou no conjunto dos números naturais em sua resposta. A resposta apresentada pelo estudante não apresentou ideias claras de nenhum dos Mundos da Matemática, mas, pensando que sua construção, pode ter sido baseada na mera visualização gráfica, acredita-se que pode apresentar características do Mundo Conceitual Corporificado.

A9: Para todo $x$ pertencente ao intervalo dado há limites, porém, quando $x$ tende a 4 , não há limite, já que os limites laterais não são iguais, isto é: $\lim _{x \rightarrow 4^{-}} f(x) \neq \lim _{x \rightarrow 4^{+}} f(x) \Rightarrow$ $\lim _{x \rightarrow 4} f(x) \nexists$.

Também A9 teve sua resposta classificada como incorreta, pois não apontou qualquer dos pontos em que não há o limite definido no intervalo dado. Percebe-se também que ficam 
evidenciadas características do Mundo Conceitual Corporificado ao apresentar sua justificativa baseada nos limites laterais.

A terceira questão tinha o seguinte enunciado:

Questão 3. Prove pela definição que $\lim _{x \rightarrow-2}(2 x+1)=-3$.

Esta questão apresenta características do Mundo Axiomático Formal e esperava-se que o aluno desenvolvesse a resposta testando um valor para $\delta$ e, após alguns cálculos, chegasse a $\delta=\frac{\varepsilon}{2}$ e continuasse com uma apresentação formal próxima a que está indicada a seguir:

$$
\begin{gathered}
|x-2|<\delta=>|x-2| \frac{\varepsilon}{2}=>2|x-2|<\varepsilon=>|2 x-4|<\varepsilon=>|(2 x+1)+3|<\varepsilon \\
\operatorname{Logo}, \forall \varepsilon>0 \exists \delta>0, \delta=\frac{\varepsilon}{2}, /|x-2|<\delta=>|(2 x+1)+3|<\varepsilon
\end{gathered}
$$

No entanto, A1, A2, A8, A10 e A12 deixaram a questão em branco, e A5, A6 e A7 declararam não lembrar a definição.

A11, A13 e A14 mostraram apenas a substituição de x por -2 e, fazendo o cálculo, concluíram que o valor do limite é -3. Talvez não tenham trabalhado, em aula, a definição formal e o uso de "épsilons e deltas" para fazer a demonstração, o que os levou a considerar que a "prova" é o cálculo; assim, julga-se que apenas os aspectos simbólicos estão presentes nessas respostas.

Os alunos A3 e A9 apresentaram uma prova, ou seja, usaram o formalismo esperado, mas trocaram hipótese por tese, porque partiram da desigualdade $|(2 x+1)-(-3)|<\varepsilon \mathrm{e}$ chegaram a $|x-2|<\delta$, usando, em todo o desenvolvimento, o símbolo de equivalência, o que é incorreto, pois a proposição envolve uma implicação.

Já o aluno A4 parece ter ideia do que seja uma demonstração formal, mas não sabe o que fazer, como se pode concluir a partir da Figura 8.

Figura 8 - Resposta de A4 à terceira questão

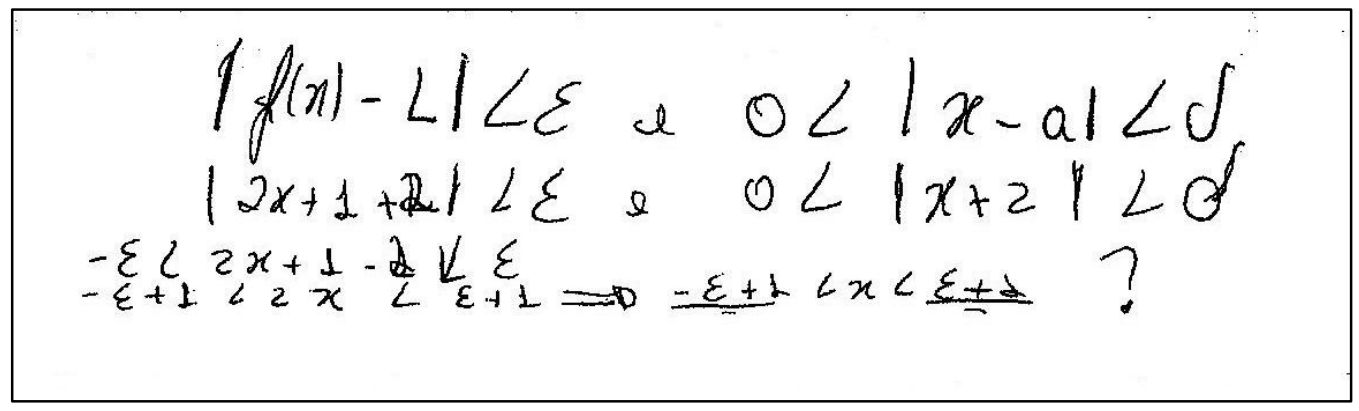

Fonte: Dados da pesquisa 
Mesmo que essa resposta apresente características do Mundo Formal, talvez os conhecimentos anteriores de A4, sobre os procedimentos para trabalhar com desigualdades que envolvem aspectos do Mundo Simbólico, não tenham sido desenvolvidos.

\section{CONSIDERAÇÕES FINAIS}

Nos cursos de Licenciatura em Matemática, são trabalhados os principais conceitos relacionados ao Cálculo Diferencial e Integral, sendo o conceito de limite um dos primeiros apresentados. Entretanto, as respostas dos estudantes às questões evidenciaram que suas aprendizagens se baseiam principalmente nas ideias desenvolvidas no âmbito do Mundo Corporificado.

Das três questões apresentadas nessa pesquisa, os alunos tiveram dificuldades em relacionar as ideias dos Mundos Simbólico e Formal com as ideias do Mundo Corporificado. Como visto na segunda questão, as conclusões a que os alunos chegam são baseadas apenas na visualização, sendo que outros argumentos simbólicos poderiam ser utilizados para dar suporte a essas resoluções. Além disso, ao se depararem com uma questão que envolvia o desenvolvimento de características do Mundo Formal, identificou-se uma grande dificuldade por parte dos estudantes em resolvê-la. Os três alunos que iniciaram o desenvolvimento da questão de uma maneira correta acabaram se perdendo em ideias do Mundo Simbólico, no trato com as desigualdades.

Dessa forma, corroborando com as ideias apresentadas quanto aos desafios do ensino de Cálculo na formação de professores de Matemática, pode-se visualizar que os alunos possuem imagens conceituais fracas no que se refere ao trabalho com questões de limites de uma função, e, principalmente, de dificuldade de realizar uma integração de seus conhecimentos em diferentes "mundos": algébrico, visual e formal. Logo, há a necessidade de um trabalho cada vez mais diverso com os conteúdos matemáticos a fim de evitar apropriações conceituais deficientes.

Percebe-se, assim, ratificando o já apresentado em Soares e Cury (2017b), que a aprendizagem do conceito de limite não está bem consolidada pelos estudantes. Certamente, o ensino desse conceito em contextos universitários precisa ser revisto. Mais do que isso, se faz necessário priorizar o trabalho com questões que fujam da mera aplicação de um algoritmo para a realização do cálculo de um limite de uma função polinomial em um ponto determinado. Além disso, é necessário focar mais na aprendizagem do conceito em si e nas suas aplicações em 
situações diversificadas, para que as experiências em sala de aula deem sentido aos conceitos aprendidos.

\section{REFERÊNCIAS}

ANTON, Howard; BIVENS, Irl; DAVIS, Stephen. Cálculo. 8. ed. Porto Alegre: Bookman, 2007.

ARTIGUE, Michèle. La enseñanza de los princípios del cálculo: problemas epistemológicos, cognitivos y didácticos. In: ARTIGUE, M. et. al. Ingeniería Didáctica em Educación Matemática. Bogotá: Grupo Editorial IberoAmérica, 1995. p. 97-140.

BARUFI, Maria Cristina Bonomi A Construção/negociação de significados no curso universitário inicial de Cálculo Diferencial e Integral. 1999. Tese (Doutorado em Educação) - Universidade de São Paulo, São Paulo, 1999. Disponível em: < http://www.teses.usp.br/teses/disponiveis/48/48133/tde-06022004-105356/pt-br.php>. Acessado em: 31 jan. 2018.

BRASIL. Conselho Nacional de Educação. Parecer 1.302/2001. Institui Diretrizes Curriculares Nacionais para os Cursos de Matemática, Bacharelado e Licenciatura. Brasília, 2001. Disponível em: 〈http://portal.mec.gov.br/cne/arquivos/pdf/CES13022.pdf〉. Acessado em 02 nov. 2017.

CURY, Helena Noronha; BAZZO, Walter Antonio. Formação crítica em Matemática: uma questão curricular? Bolema, Rio Claro, v. 14, n. 16, p. 29-47, 2001. Disponível em: < http://ojs-teste.biblioteca.unesp.br/index.php/bolema/article/view/10615>. Acessado em: 05 fev. 2019.

LOPES, Artur Oscar. Algumas reflexões sobre a questão do alto índice de reprovação nos cursos de Cálculo da UFRGS. Matemática Universitária, Rio de Janeiro, n. 26/27, p. 123 146, 1999. Disponível em: <https://rmu.sbm.org.br/wpcontent/uploads/sites/27/2018/03/n26_n27_Artigo05.pdf>. Acessado em: 11 fev. 2019.

MEYER, João Frederico da Costa Azevedo, SOUZA JUNIOR. Arlindo José de. A utilização do computador no processo de ensinar-aprender Cálculo: a constituição de grupos de ensino com pesquisa no interior da universidade. Zetetiké, Campinas, v.10, n. 17/18, p. 113-148, jan./dez. 2002. Disponível em:

<https://periodicos.sbu.unicamp.br/ojs/index.php/zetetike/article/view/8646945>. Acessado em: 11 fev. 2019.

SENA, Thainnã Thatisuane Oliveira; SOUZA, Ademária Aparecida. Causas de dificuldades no ensino-aprendizagem de Cálculo Diferencial e Integral na perspectiva dos alunos e dos professores do curso de Matemática da UFAL - Campus de Arapiraca. Proceeding Series of the Brazilian Society of Applied and Computational Mathematics, São Carlos, v. 3, n. 1, p. 1-2, 2015. Disponível em: 〈https://proceedings.sbmac.org.br/sbmac/article/view/807/813>. Acessado em: 28 fev. 2019.

SOARES, Gabriel de Oliveira; CURY, Helena Noronha. As ideias de David Tall em um mapeamento de artigos de periódicos brasileiros. Revista Sergipana de Matemática e 
Educação Matemática, n. 1, p. 1-16, 2017a. Disponível em:

<https://seer.ufs.br/index.php/ReviSe/article/view/7294>. Acessado em: 01 fev. 2019.

SOARES, Gabriel de Oliveira; CURY, Helena Noronha. O conteúdo de limite em cursos de licenciatura em Matemática: uma pesquisa à luz da teoria dos três mundos da Matemática.

ReBECEM, v.1, n. 1, p. 64-83, 2017b. Disponível em: 〈http://erevista.unioeste.br/index.php/rebecem/article/view/18557/121967 . Acessado em: 15 fev. 2019.

STARON, Francielly. O monstro da reprovação em Cálculo Diferencial Integral. In: Conversando sobre extensão, 14., 2016, Ponta Grossa. Anais... Ponta Grossa, 2016. p. 1-7. Disponível em: <http://sites.uepg.br/conex/anais/anais_2016/anais2016/1329-4673-1-DRmod.pdf>. Acessado em: 08 fev. 2019.

TALL, David. How humans learn to think mathematically. Cambridge: Cambridge University Press, 2013.

TALL, David. Thinking through three worlds of mathematics. In: INTERNATIONAL CONFERENCE FOR THE PSYCHOLOGY OF MATHEMATICS EDUCATION, 28., 2004, Bergen, Norway. Proceedings... Bergen: PME, 2004. p. 281-288.

ZUCHI, Ivanete; GONÇALVES, Mirian Buss. Investigação sobre os obstáculos de aprendizagem do conceito de limite. In: CONGRESSO BRASILEIRO DE ENSINO DE ENGENHARIA, 31., 2003, Rio de Janeiro. Anais... Rio de Janeiro: ABENGE, 2003. Disponível em: 〈http://www.abenge.org.br/cobenge/arquivos/16/artigos/NMT210.pdf> . Acessado em: 08 fev. 2019.

Recebido em: 1 de março de 2019.

Aprovado em: 25 de junho de 2019. 